\title{
Change in non-small-cell lung cancer tumor size in patients treated with nintedanib plus docetaxel: analyses from the Phase III LUME-Lung I study
}

This article was published in the following Dove Press journal:

OncoTargets and Therapy

\author{
Martin Reck' \\ Anders Mellemgaard ${ }^{2}$ \\ Silvia Novello ${ }^{3}$ \\ Pieter E Postmus ${ }^{4}$ \\ Birgit Gaschler-Markefski ${ }^{5}$ \\ Rolf Kaiser ${ }^{5,6}$ \\ Hannes Buchner ${ }^{7}$ \\ 'Department of Thoracic Oncology, \\ Lung Clinic Grosshansdorf, Airway \\ Research Center North (ARCN), \\ Member of the German Center for \\ Lung Research (DZL), Grosshansdorf, \\ Germany; ${ }^{2}$ Department of Internal \\ Medicine and Oncology, Bornholms \\ Hospital, Ronne, Denmark; \\ ${ }^{3}$ Department of Oncology, University \\ of Turin, S. Luigi Hospital, Torino, Italy; \\ ${ }^{4}$ Leiden University Medical Center, \\ Leiden, the Netherlands; ${ }^{5}$ Boehringer \\ Ingelheim Pharma GmbH \& Co. KG, \\ Biberach an der Riss, Germany; \\ ${ }^{6}$ Institute of Pharmacology, University \\ Medical Center, Johannes Gutenberg \\ University Mainz, Mainz, Germany; \\ ${ }^{7}$ Staburo GmbH, Munich, Germany
}

Correspondence: Martin Reck Department of Thoracic Oncology, Lung Clinic Grosshansdorf, Airway Research Center North (ARCN), Member of the German Center for Lung Research (DZL), Wöhrendamm 80, 22927

Grosshansdorf, Germany

Tel +494 I02 601 2101

Fax +49 4 I02 60। 7।0।

Email dr.martin.reck@web.de
Background: Nintedanib in combination with docetaxel is approved in the European Union and other countries for the treatment of patients with advanced non-small-cell lung cancer (NSCLC) of adenocarcinoma histology after first-line chemotherapy, based on the overall survival findings of Phase III LUME-Lung 1 study. Change in target lesion size over time as a treatment effect was assessed in patients from this study.

Methods: Tumor size was evaluated using predefined tumor measurements. Mixed-effects models were used to quantify individual relationships between time from randomization and tumor burden, measured as the sum of longest diameter (SLD) of target lesions and assessed by an independent review (Response Evaluation Criteria In Solid Tumors [RECIST] v1.0). Exploratory analyses were conducted on the overall adenocarcinoma population, adenocarcinoma patients with time from start of first-line therapy $<9$ months (TSFLT $<9$ ), adenocarcinoma patients who had progressive disease as best response to first-line therapy (PD-FLT), and in squamous cell carcinoma patients.

Results: Estimated mean baseline SLD was $82.5 \mathrm{~mm}$ in the adenocarcinoma ( $\mathrm{n}=658), 88.3 \mathrm{~mm}$ in the TSFLT $<9(\mathrm{n}=405), 98.1 \mathrm{~mm}$ in the PD-FLT $(\mathrm{n}=117)$, and $94.3 \mathrm{~mm}$ in the squamous cell carcinoma $(n=555)$ populations. Treatment with nintedanib/docetaxel showed a significant reduction in tumor size over time $(P<0.0001)$ in patients with adenocarcinoma compared with placebo/docetaxel, and in patients with squamous cell carcinoma $(P=0.0049)$. Treatment difference at 6 months was $9.7 \mathrm{~mm}$ in the overall adenocarcinoma population, $16.8 \mathrm{~mm}$ in the TSFLT $<9$ population, $19.7 \mathrm{~mm}$ in the PD-FLT population, and $6.8 \mathrm{~mm}$ in the squamous cell carcinoma population. SLD at 2 months post-randomization was identified as a surrogate endpoint for overall survival, in addition to progression-free survival, for all except the PD-FLT population.

Conclusion: Treatment with nintedanib/docetaxel significantly decreased tumor burden and decelerated tumor size over time compared with placebo/docetaxel in the overall adenocarcinoma population, including in patients with the poorest prognosis due to aggressive tumor dynamics.

Keywords: adenocarcinoma, non-small-cell lung cancer, sum of longest diameters, surrogate OS endpoints, squamous cell carcinoma, tumor burden

\section{Introduction}

The ultimate aim of any anticancer treatment is to reduce progressive disease (PD) and prolong overall survival (OS) of patients. ${ }^{1,2}$ Measurement of tumor burden has been shown to be associated with clinical outcomes in advanced non-small-cell lung cancer (NSCLC); ${ }^{3,4}$ when measured by the sum of longest diameter (SLD) of target lesions, baseline tumor burden is a predictor of survival duration..$^{3,4}$ Tumor growth rate is an 
independent prognostic factor for patients with lung cancer. ${ }^{5}$ Measuring tumor volume changes (eg, tumor size rate, time to tumor size, early tumor shrinkage, SLD at specific time points, depth of tumor response/nadir) during treatment of different tumor types, and in multiple treatment settings, provides a quantitative and dynamic evaluation of tumor response that can potentially identify early treatment sensitivity and has been correlated with survival outcomes., ${ }^{4,-12}$

The randomized, double-blind, placebo-controlled, Phase III LUME-Lung 1 study (ClinicalTrials.gov NCT00805194; 1199.13), comparing the triple angiokinase inhibitor nintedanib in combination with docetaxel with placebo/docetaxel, demonstrated a significant improvement in OS in patients with NSCLC of adenocarcinoma histology after failure of first-line chemotherapy (median OS: 12.6 vs 10.3 months; hazard ratio [HR]: 0.83 [95\% CI: $0.70-0.99] ; P=0.0359) .{ }^{13}$ A correlation between OS benefit from nintedanib treatment and the continuous variable "time from start of first-line treatment" (TSFLT) has been observed. ${ }^{13,14}$ In adenocarcinoma patients with TSFLT $<9$ months (TSFLT $<9$ ), OS was significantly longer in the nintedanib arm than in the placebo arm, with a 3.0-month improvement (median OS 10.9 vs 7.9 months; HR: 0.75 [95\% CI: 0.60-0.92]; $P=0.0073) .{ }^{13}$

The outcomes of the LUME-Lung 1 study contributed to the approval of nintedanib in the European Union and other countries for the treatment of patients with locally advanced, metastatic, or locally recurrent NSCLC of adenocarcinoma histology after first-line chemotherapy. ${ }^{15}$

In the LUME-Lung 1 study, predefined sensitivity analyses of OS used the prognostic factor of baseline SLD of target lesions as an adjustment for baseline tumor burden. When the model was adjusted for the baseline SLD, OS remained significantly improved with nintedanib/docetaxel in the overall adenocarcinoma population and in adenocarcinoma patients with TSFLT $<9$, and was significant even in all patients. In patients with squamous cell carcinoma, there was no difference in OS (HR: 1.01 [95\% CI: $0.85-1.21$ ]; $P=0.8907$ ) in the primary analysis; however, adjustment for SLD resulted in a notable decrease in the HR to 0.92 (95\% CI: 0.77-1.10; $P=0.365)$. Overall, a greater tumor burden was associated with a greater treatment effect in the nintedanib arm. ${ }^{13,16}$

These observations suggest that tumor characteristics, including tumor burden and the dynamics of PD, may have predictive potential for nintedanib therapy. ${ }^{17}$ The current investigation extends the analyses of tumor burden. We explored the impact of tumor size over time, including the identification of surrogate endpoints of survival, as well as the time to occurrence of new lesions as a treatment effect in patients from the Phase III LUME-Lung 1 study, with a specific focus on patients with a poorer prognosis due to more aggressive tumors.

\section{Methods}

\section{Study design and assessments}

Details of the LUME-Lung 1 study design and assessments have been reported previously ${ }^{13}$ and are briefly summarized below. Patients in the randomized, double-blind, placebocontrolled, Phase III LUME-Lung 1 study received standard intravenous docetaxel $\left(75 \mathrm{mg} / \mathrm{m}^{2}\right)$ on Day 1 and nintedanib (200 mg twice daily; nintedanib arm) or matching placebo (placebo arm) on Days 2-21, until PD or non-tolerability. ${ }^{13}$ Target lesions were assessed by central independent review using modified Response Evaluation Criteria In Solid Tumors (RECIST) v1.0 at baseline and every 6 weeks after the first administration of docetaxel.

All patients provided written informed consent, and the study protocol was approved by independent ethics committees or institutional review boards at each center (Table S1). Here, we specifically report exploratory analyses for the overall adenocarcinoma population, the population of patients with adenocarcinoma and TSFLT $<9$, and adenocarcinoma patients with PD as their best response to first-line therapy (PD-FLT), as well as the population with squamous cell carcinoma.

Efficacy outcomes for this study have been previously reported. ${ }^{13,18}$ Treatment with nintedanib/docetaxel significantly improved efficacy outcomes in the analyzed adenocarcinoma populations (Table S2).

\section{Assessment of tumor size}

Tumor size was evaluated using all available tumor measurements from patients who had at least two evaluable assessments. Tumor burden was measured as the SLD of target lesions, assessed by an independent central review (RECIST v1.0) preferentially, although data from investigator assessment (if available) were used for patients without two evaluable central independent review measurements.

\section{New lesions}

The time to occurrence of new lesions, as recorded by RECIST v1.0, was analyzed using a Cox model, KaplanMeier curves, and a log-rank test, similar to the primary analysis. Two exploratory sensitivity analyses were carried out: first, patients were recorded as being censored using the OS results (time to new lesion) and, second, death was counted as an event and patients could have either an event or be censored using the OS results (new lesion-free survival). 


\section{Surrogate endpoints for OS}

Variables proposed in literature related to tumor size over time were evaluated for their predictive value for OS. These variables, together with baseline characteristics, were included in the stepwise algorithm to determine surrogate endpoints for OS, while additionally adjusting for important prognostic baseline variables. Baseline characteristics were investigated for being predictive for OS. The baseline characteristics included the trial's stratification factors (Eastern Cooperative Oncology Group performance status [0 vs 1], prior receipt of bevacizumab [yes vs no], presence of brain metastases at baseline [yes vs no]), as well as other characteristics that were predefined in the protocol (sex [female vs male], age [ $<65$ vs $\geq 65$ years], geographic region, race category [Asian vs non-Asian], smoking status [current smoker vs ex-smoker/never smoked], use of bisphosphonates at baseline [yes vs no], best response to first-line therapy [complete response/partial response/stable disease vs PD], number of metastatic organs [ $\leq 2 \mathrm{vs}>2$ ], adrenal metastases [yes vs no], liver metastases [yes vs no], and lactate dehydrogenase at baseline [ $>1$ vs $\leq 1$ upper limit of normal]), TSFLT, and SLD of target lesions.

Based on previous clinical methodology and in accordance with RECIST recommendations, these variables related to tumor size included the "absolute reduction in SLD from baseline to 2 months after randomization"; 19,20 "percentage reduction in SLD from baseline to 2 months after randomization"; "SLD at 2 months after randomization"; "maximum reduction in SLD compared to baseline"; "maximum reduction in percentage of SLD compared to baseline"; "SLD at nadir (lowest tumor burden after randomization)"; "estimated SLD at baseline"; "time to tumor growth (time to nadir)" (Figure S1);,22,23 "early tumor shrinkage" analyzed both as any tumor increase (but without new metastases) and as minor shrinkage (both analyses took "new metastases" as the reference population), $7,7,10$ "time to return to baseline SLD"; "observed SLD at baseline",; "progressionfree survival (PFS) duration", including patients who were censored in the analysis of PFS; and "PFS flag", referring to the patients who were censored in the analysis of PFS. These censored patients were compared with patients who had a PFS event (censoring vs event).

\section{Statistical analyses and use of surrogate endpoints}

Mixed-effects models were used to quantify the nonlinear individual relationships between time from randomization $(t)$ and tumor burden. Several models were evaluated and the model that performed best in terms of Akaike Information
Criterion (a measure of the relative quality of statistical models) was used: $\operatorname{SLD}(\mathrm{t}) \mathrm{i}=$ beta $1 * \mathrm{t}+$ beta $2 * \ln (\mathrm{t}+1)+\mathrm{b} 1 \mathrm{i}^{*} \mathrm{t}+$ $b 2 i * \ln (t+1)$, where $i=1, \ldots, n, n=$ number of patients, and b1i and $b 2 \mathrm{i}$ are random effects. The selected model was flexible, allowing for a wide range of differently shaped growth curves (U-shaped, J-shaped, N-shaped, and linear-shaped). Cox regression and Kaplan-Meier plots were used to evaluate and visualize the predictive value of surrogate endpoints. A stepwise selection method was used, with inclusion and exclusion significance of 0.1 .

\section{Results}

\section{Patient population}

In the LUME-Lung 1 study, there were 658 patients with adenocarcinoma histology. Of these, 405 had TSFLT $<9$ and 117 had PD-FLT. In addition, there were 555 patients with squamous cell carcinoma. Most patients had metastatic cancer at screening; this included $94.2 \%$ of the overall adenocarcinoma population, $96.8 \%$ of the TSFLT $<9$ adenocarcinoma population, $95.7 \%$ of the PD-FLT adenocarcinoma population, and $86.3 \%$ of the squamous cell carcinoma population (Table 1). Approximately half the patients had one or two metastatic sites at screening (Table S3). In the overall adenocarcinoma population, the median duration of treatment with nintedanib/docetaxel was 4.2 months (range $0-41.5$ ) and with placebo/docetaxel was 3.0 months (range $0-31.7$ ), while in the squamous cell carcinoma population, the median duration of treatment with nintedanib/docetaxel was 2.8 months (range $0-34.9$ ) and with placebo/docetaxel was 2.5 months (range $0-35.6$ ).

\section{Tumor evaluation and baseline tumor SLD}

A summary of radiologic assessments and baseline SLD is shown in Table 2. Most patients had at least one valid post-baseline image taken by computed tomography or a magnetic resonance imaging scan, with the same method used consistently for an individual patient $(93.3 \%$ of the overall adenocarcinoma population, $92.3 \%$ of the TSFLT $<9$ adenocarcinoma population, $89.7 \%$ of the PD-FLT adenocarcinoma population, and $90.5 \%$ of the squamous cell carcinoma population). The estimated mean baseline SLD measurements were larger in the population with the worse prognosis (PD-FLT).

\section{Tumor size over time}

Estimated tumor size curves for the adenocarcinoma populations evaluated in this study are shown in Figure 1. Tumor size patterns were consistent between treatment arms in all adenocarcinoma populations. For adenocarcinoma patients 
Table I Metastatic sites at screening

\begin{tabular}{|c|c|c|c|c|c|c|c|c|}
\hline & \multicolumn{6}{|c|}{ Patients with adenocarcinoma histology NSCLC } & \multirow{2}{*}{\multicolumn{2}{|c|}{$\begin{array}{l}\text { Patients with } \\
\text { squamous cell } \\
\text { histology NSCLC }\end{array}$}} \\
\hline & \multicolumn{2}{|l|}{ All } & \multicolumn{2}{|l|}{ TSFLT $<9$} & \multicolumn{2}{|l|}{ PD-FLT } & & \\
\hline & $\begin{array}{l}\text { Nintedanib } \\
(n=322)\end{array}$ & $\begin{array}{l}\text { Placebo } \\
(n=336)\end{array}$ & $\begin{array}{l}\text { Nintedanib } \\
(\mathrm{n}=206)\end{array}$ & $\begin{array}{l}\text { Placebo } \\
(n=199)\end{array}$ & $\begin{array}{l}\text { Nintedanib } \\
(n=53)\end{array}$ & $\begin{array}{l}\text { Placebo } \\
(n=64)\end{array}$ & $\begin{array}{l}\text { Nintedanib } \\
(n=276)\end{array}$ & $\begin{array}{l}\text { Placebo } \\
(n=279)\end{array}$ \\
\hline Patients with metastases at screening, n (\%) & $300(93.2)$ & $320(95.2)$ & $197(95.6)$ & $195(98.0)$ & $50(94.3)$ & $62(96.9)$ & $235(85.1)$ & $244(87.5)$ \\
\hline \multicolumn{9}{|l|}{ Metastatic sites at screening, n (\%) } \\
\hline 0 & $22(6.8)$ & $16(4.8)$ & $9(4.4)$ & $4(2.0)$ & $3(5.7)$ & $2(3.1)$ & $4 \mid(14.9)$ & $35(12.5)$ \\
\hline $1-2$ & $187(58.1)$ & $196(58.3)$ & $116(56.3)$ & II $3(56.8)$ & $27(50.9)$ & $34(53.1)$ & $164(59.4)$ & $176(63.1)$ \\
\hline$>2$ & $113(35.1)$ & $124(36.9)$ & $81(39.3)$ & $82(4 I .2)$ & $23(43.4)$ & $28(43.8)$ & $71(25.7)$ & $68(24.4)$ \\
\hline \multicolumn{9}{|c|}{ Location of metastatic sites at screening, $n(\%)$} \\
\hline Lung ipsilateral & $163(50.6)$ & $175(52.1)$ & $109(52.9)$ & $103(51.8)$ & $30(56.6)$ & $34(53.1)$ & $105(38.0)$ & II $5(4 \mid .2)$ \\
\hline Lung contralateral & $140(43.5)$ & $144(42.9)$ & $87(42.2)$ & $83(41.7)$ & $25(47.2)$ & $29(45.3)$ & $98(35.5)$ & $90(32.3)$ \\
\hline Bone & $92(28.6)$ & $100(29.8)$ & $66(32.0)$ & $73(36.7)$ & $18(34.0)$ & $22(34.4)$ & $43(15.6)$ & $48(17.2)$ \\
\hline Liver & $63(19.6)$ & $53(15.8)$ & $45(21.8)$ & $35(17.6)$ & $14(26.4)$ & $13(20.3)$ & $64(23.2)$ & $45(16.1)$ \\
\hline Adrenal glands & $42(13.0)$ & $56(16.7)$ & $29(14.1)$ & $40(20.1)$ & $8(15.1)$ & $15(23.4)$ & $36(13.0)$ & $45(16.1)$ \\
\hline Brain & $26(8.1)$ & $23(6.8)$ & $14(6.8)$ & $10(5.0)$ & $5(9.4)$ & $2(3.1)$ & $6(2.2)$ & II (3.9) \\
\hline Other & 138 (42.9) & I 56 (46.4) & $94(45.6)$ & $104(52.3)$ & $26(49.1)$ & $34(53.1)$ & $124(44.9)$ & $129(46.2)$ \\
\hline
\end{tabular}

Abbreviations: NSCLC, non-small-cell lung cancer; PD-FLT, progressive disease as the best response to first-line therapy; TSFLT, time from start of first-line therapy $<9$ months.

treated with nintedanib/docetaxel, a nonlinear, J-shaped curve was observed, indicating a decline in SLD at the beginning of treatment. This trend was maintained over time and followed by a linear increase in SLD. In contrast, for adenocarcinoma patients treated with placebo/docetaxel, a linear increase in SLD from baseline over time was observed.

In the overall adenocarcinoma population, disease control was achieved in $60.2 \%$ of patients in the nintedanib arm and $44.0 \%$ in the placebo arm. ${ }^{13}$ At baseline, the estimated slope of the tumor size curve was -5.80 in the nintedanib arm and
1.93 in the placebo arm. In the nintedanib arm, the tumor size curve nadir was 1.34 and the time when the average SLD values returned to baseline was 3-4 months (Figure 1A). Six months after randomization, the difference between the two treatment arms in the estimated mean SLD measurements was $9.7 \mathrm{~mm}(88.7 \mathrm{~mm}$ in the nintedanib arm vs $98.4 \mathrm{~mm}$ in the placebo arm).

In all adenocarcinoma patient populations, treatment with nintedanib/docetaxel produced a significant reduction in tumor size over time compared with placebo/docetaxel in

Table 2 Radiological assessments and baseline SLD of target lesions

\begin{tabular}{|c|c|c|c|c|c|c|c|c|}
\hline & \multicolumn{6}{|c|}{ Patients with adenocarcinoma histology NSCLC } & \multirow{2}{*}{\multicolumn{2}{|c|}{$\begin{array}{l}\text { Patients with } \\
\text { squamous cell } \\
\text { histology NSCLC }\end{array}$}} \\
\hline & \multicolumn{2}{|c|}{ All } & \multicolumn{2}{|c|}{ TSFLT $<9$} & \multicolumn{2}{|l|}{ PD-FLT } & & \\
\hline & $\begin{array}{l}\text { Nintedanib } \\
(n=322)\end{array}$ & $\begin{array}{l}\text { Placebo } \\
(n=336)\end{array}$ & $\begin{array}{l}\text { Nintedanib } \\
(n=206)\end{array}$ & $\begin{array}{l}\text { Placebo } \\
(n=199)\end{array}$ & $\begin{array}{l}\text { Nintedanib } \\
(n=53)\end{array}$ & $\begin{array}{l}\text { Placebo } \\
(n=64)\end{array}$ & $\begin{array}{l}\text { Nintedanib } \\
(n=276)\end{array}$ & $\begin{array}{l}\text { Placebo } \\
(n=279)\end{array}$ \\
\hline $\begin{array}{l}\text { Patients with at least } \\
\text { one valid post-baseline } \\
\text { image, }{ }^{a} \mathrm{n}(\%)\end{array}$ & $306(95.0)$ & $308(91.7)$ & $196(95.1)$ & $178(89.4)$ & $49(92.5)$ & $56(87.5)$ & $252(91.3)$ & $250(89.6)$ \\
\hline $\begin{array}{l}\text { Number of valid post- } \\
\text { baseline images }\end{array}$ & 1,273 & 1,125 & 769 & 513 & 230 & 149 & 843 & 737 \\
\hline $\begin{array}{l}\text { Number of valid post- } \\
\text { baseline images per } \\
\text { patient, median (range) }\end{array}$ & $3(I-28)$ & $3(|-2|)$ & $3(I-2 I)$ & $2(I-20)$ & $3(I-2 I)$ & $2(1-13)$ & $2(I-24)$ & $2(I-24)$ \\
\hline $\begin{array}{l}\text { Estimated mean } \\
\text { (range) baseline SLD } \\
\text { measurements }(\mathrm{mm})\end{array}$ & \multicolumn{2}{|c|}{$82.5(10.9-391.2)$} & \multicolumn{2}{|c|}{$88.3(11.2-391.2)$} & \multicolumn{2}{|c|}{$98.1(11.5-391.2)$} & \multicolumn{2}{|c|}{$94.3(11.3-313.9)$} \\
\hline
\end{tabular}

Notes: ${ }^{a}$ Either CT or MRI scan, with the same method used consistently for an individual patient. ${ }^{b}$ Estimated fixed effects of longitudinal mixed model; the intercept of SLD was at Month 0 .

Abbreviations: CT, computed tomography; MRI, magnetic resonance imaging; NSCLC, non-small-cell lung cancer; PD-FLT, progressive disease as the best response to first-line therapy; SLD, sum of longest diameter; TSFLT $<9$, time from start of first-line therapy $<9$ months. 

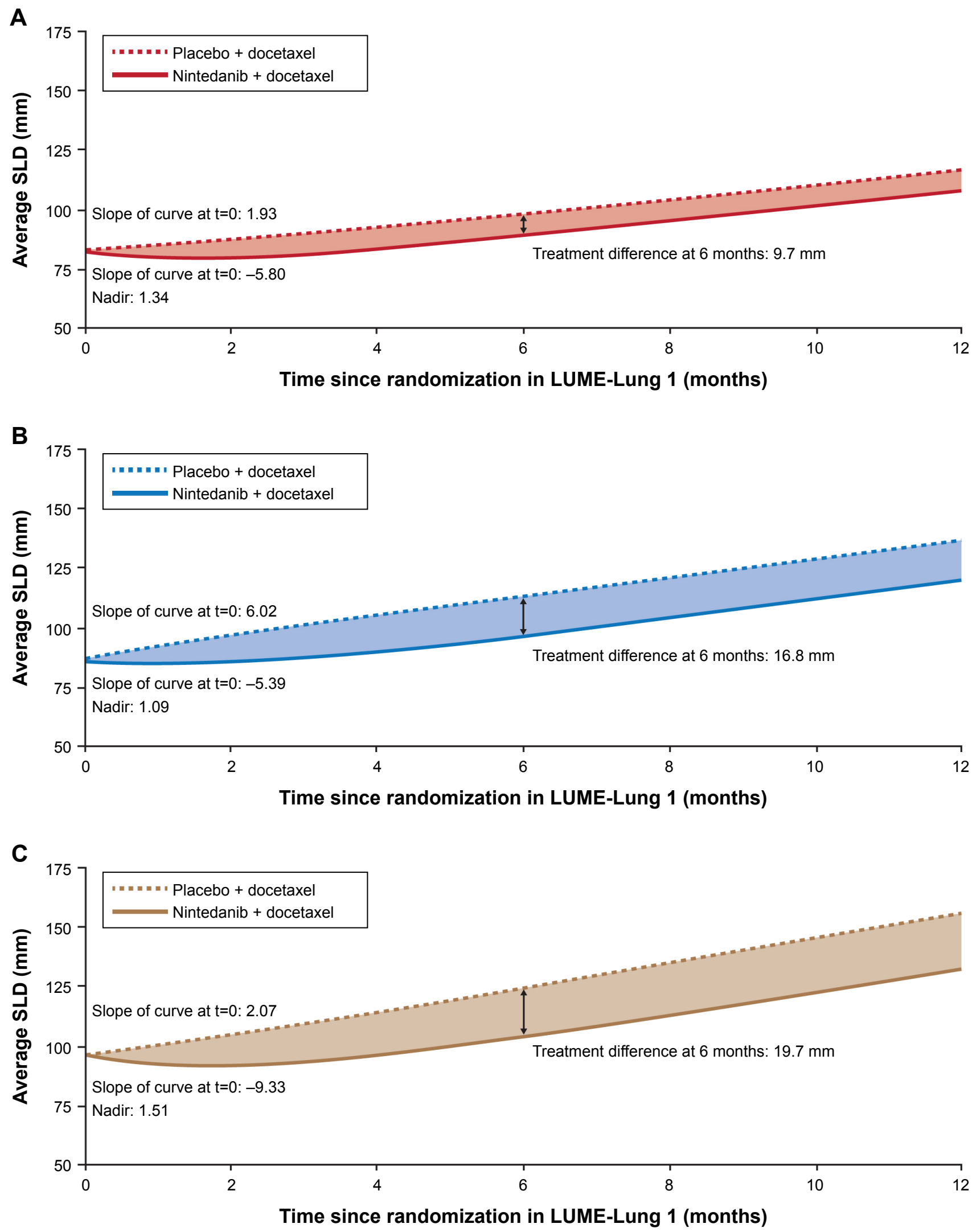

Figure I Tumor size over time, measured by SLD of target lesions, in patients with adenocarcinoma.

Notes: Trajectories are based on the estimated fixed effects of the mixed model. (A) All patients with adenocarcinoma. (B) Patients with adenocarcinoma who progressed within 9 months after start of first-line therapy (TSFLT <9). (C) Patients with adenocarcinoma with PD-FLT.

Abbreviations: PD-FLT, progressive disease as the best response to first-line therapy; SLD, sum of longest diameter; $t$, time from randomization; TSFLT <9, time from start of first-line therapy $<9$ months. 


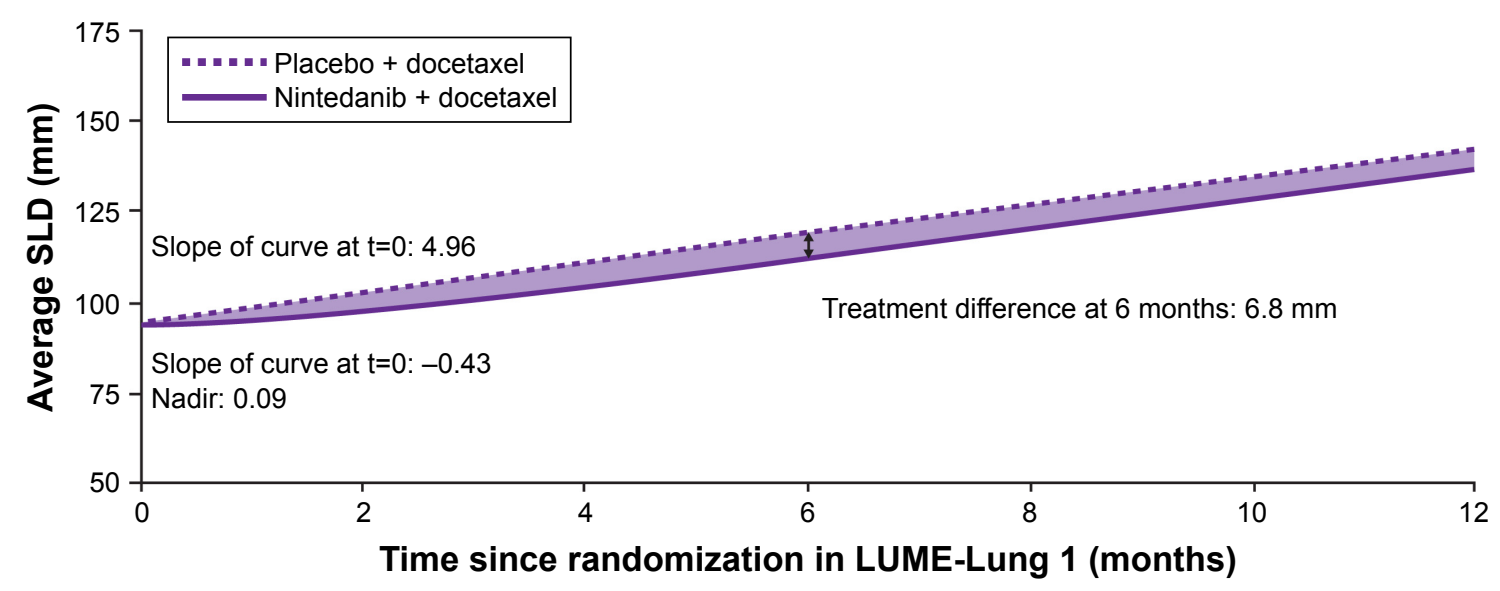

Figure 2 Tumor size over time, measured by SLD of target lesions, in patients with squamous cell carcinoma.

Note: Trajectories are based on the estimated fixed effects of the mixed model.

Abbreviations: SLD, sum of longest diameter; $t$, time from randomization.

the likelihood ratio test of the treatment effect $(P<0.0001$ for the overall adenocarcinoma population). The treatment difference at 6 months was even more pronounced in patients with a poor prognosis $(P<0.0001$ for the TSFLT $<9$ adenocarcinoma population; $P=0.0004$ for the PD-FLT adenocarcinoma population), as shown in Figure 1B and C.

In all groups, nintedanib/docetaxel produced a significant reduction in tumor size over time compared with placebo/ docetaxel. Disease stabilization was greater in patients receiving nintedanib than in patients receiving placebo (Table S2). Following the same trend, in squamous cell carcinoma patients, treatment with nintedanib/docetaxel produced a significant reduction in tumor size over time compared with placebo ( $P=0.0049$; Figure 2). Further estimates for the parameterization of the longitudinal model for tumor size are reported in Table S4.

Throughout the whole study, there was a sustained difference in the average SLD of target lesions between the two arms, with lower tumor burden in the nintedanib arm (Figure 3).

\section{New lesions}

The analysis of the time to occurrence of new lesions favored the nintedanib arm and in the second sensitivity analysis was significantly longer with nintedanib in the TSFLT $<9$ and PD-FLT populations (Table 3). The frequency for each site was mostly balanced across the two treatment arms. There was no specific pattern for the location of new lesions associated with either treatment arm (Table S5).

\section{Surrogate endpoints for OS}

Several variables related to tumor size were selected by the algorithm as surrogate endpoints for OS (Table 4) in patients with a baseline and at least one post-baseline measurement
(614 patients with adenocarcinoma, 374 patients with TSFLT $<9$ adenocarcinoma, 105 patients with PD-FLT adenocarcinoma, and 502 patients with squamous cell carcinoma). The algorithm demonstrated that PFS duration and PFS censorship were both predictive for OS in all populations evaluated, with the exception of the PD-FLT population,

\section{Time since randomization in LUME- Lung 1 (months)}

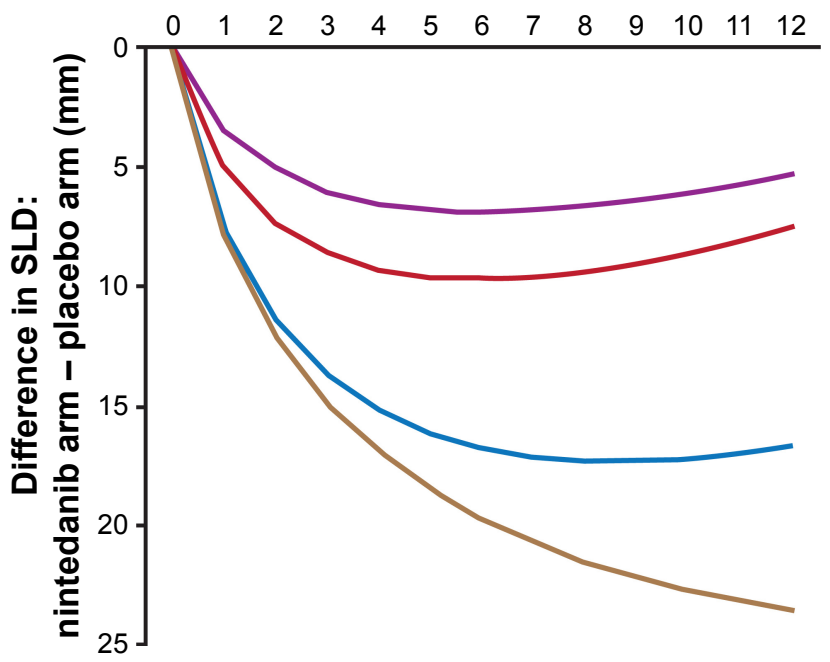

Patients with adenocarcinoma histology NSCLC (all)
Patients with adenocarcinoma histology NSCLC and
TSFLT <9 months
Patients with adenocarcinoma histology NSCLC and
PD-FLT
Patients with squamous cell histology NSCLC

Figure 3 Difference between the absolute SLD of target lesions (nintedanib arm placebo arm) at different time intervals.

Note: The degree of SLD difference shows the degree of response to treatment. Abbreviations: NSCLC, non-small-cell lung cancer; PD-FLT, progressive disease as the best response to first-line therapy; SLD, sum of longest diameter; TSFLT, time from start of first-line therapy. 
Table 3 Time to occurrence of new lesions

\begin{tabular}{|c|c|c|c|c|c|c|c|c|}
\hline & \multicolumn{6}{|c|}{ Patients with adenocarcinoma histology NSCLC } & \multirow{2}{*}{\multicolumn{2}{|c|}{$\begin{array}{l}\text { Patients with } \\
\text { squamous cell } \\
\text { histology NSCLC }\end{array}$}} \\
\hline & \multicolumn{2}{|l|}{ All } & \multicolumn{2}{|l|}{ TSFLT $<9$} & \multicolumn{2}{|l|}{ PD-FLT } & & \\
\hline & $\begin{array}{l}\text { Nintedanib } \\
(n=322)\end{array}$ & $\begin{array}{l}\text { Placebo } \\
(n=336)\end{array}$ & $\begin{array}{l}\text { Nintedanib } \\
(\mathrm{n}=206)\end{array}$ & $\begin{array}{l}\text { Placebo } \\
(n=199)\end{array}$ & $\begin{array}{l}\text { Nintedanib } \\
(n=53)\end{array}$ & $\begin{array}{l}\text { Placebo } \\
(n=64)\end{array}$ & $\begin{array}{l}\text { Nintedanib } \\
(\mathrm{n}=276)\end{array}$ & $\begin{array}{l}\text { Placebo } \\
(n=279)\end{array}$ \\
\hline \multicolumn{9}{|l|}{ Sensitivity analysis Ia } \\
\hline Patients with event, n (\%) & $142(44.1)$ & $154(45.8)$ & $89(43.2)$ & $93(46.7)$ & $19(35.8)$ & $28(43.8)$ & $106(38.4)$ & III (39.8) \\
\hline \multicolumn{9}{|c|}{ Time to occurrence of new lesions (months) } \\
\hline Median (months) & 11.7 & 10.9 & 11.3 & 9.9 & NR & 7.3 & 26.3 & 18.2 \\
\hline HR (95\% Cl); $P$-value & \multicolumn{2}{|c|}{$0.88(0.70-\mathrm{I} . \mathrm{II}) ; P=0.2748$} & \multicolumn{2}{|c|}{$0.79(0.59-1.06) ; P=0.1|8|$} & \multicolumn{2}{|c|}{$0.58(0.3 \mathrm{I}-\mathrm{I} .09) ; P=0.0848$} & \multicolumn{2}{|c|}{$0.88(0.67-I .14) ; P=0.3334$} \\
\hline \multicolumn{9}{|l|}{ Sensitivity analysis $2^{b}$} \\
\hline Patients with event, n (\%) & $285(88.5)$ & $295(87.8)$ & $187(90.8)$ & $182(9 \mid .5)$ & $49(92.5)$ & $60(93.8)$ & $259(93.8)$ & $253(90.7)$ \\
\hline New lesions & $142(44.1)$ & $154(45.8)$ & $89(43.2)$ & $93(46.7)$ & $19(35.8)$ & $28(43.8)$ & $106(38.4)$ & III (39.8) \\
\hline Death & $143(44.4)$ & $|4|(42.0)$ & $98(47.6)$ & $89(44.7)$ & $30(56.6)$ & $32(50.0)$ & $153(55.4)$ & $142(50.9)$ \\
\hline \multicolumn{9}{|c|}{ Time to occurrence of new lesions (months) } \\
\hline Median (months) & 6.9 & 5.6 & 6.4 & 4.7 & 6.0 & 4.5 & 5.6 & 4.6 \\
\hline HR (95\% Cl); $P$-value & \multicolumn{2}{|c|}{$0.90(0.76-1.06) ; P=0.1970$} & \multicolumn{2}{|c|}{$0.77(0.63-0.95) ; P=0.016 \mathrm{I}$} & \multicolumn{2}{|c|}{$0.62(0.4 \mathrm{I}-0.94) ; P=0.0232$} & \multicolumn{2}{|c|}{$0.93(0.78-I . I I) ; P=0.4295$} \\
\hline
\end{tabular}

Notes: an sensitivity analysis I, patients were recorded as being censored using the OS results (time to new lesion). bln sensitivity analysis 2, death was counted as an event and patients could have either an event or be censored using the OS results (new lesion-free survival).

Abbreviations: HR, hazard ratio; NR, not reached; NSCLC, non-small-cell lung cancer; OS, overall survival; PD-FLT, progressive disease as the best response to first-line therapy; TSFLT $<9$, time from start of first-line therapy $<9$ months.

a finding probably associated with the low numbers of censored patients in this population ( $\mathrm{n}=14$ [12\%]). Regarding SLD, a smaller SLD at 2 months was predictive for longer OS in all the populations evaluated, except for the PD-FLT population. Baseline variables that were selected as being predictive for OS can be found in Table S6.

\section{Discussion}

Our analysis showed that the nintedanib/docetaxel combination slowed down the tumor dynamics compared with placebo/docetaxel, with the largest effect seen in patients with the poorest prognosis. Corresponding well with the OS findings of LUME-Lung 1, the analyses of data on the

Table 4 Surrogate endpoints for OS (tumor size variables)

\begin{tabular}{|c|c|c|c|}
\hline Population & Variable & HR $(95 \% \mathrm{Cl})^{\mathrm{a}}$ & $P$-value ${ }^{b}$ \\
\hline \multicolumn{4}{|c|}{ Patients with adenocarcinoma histology NSCLC } \\
\hline \multirow[t]{6}{*}{ All } & SLD of target lesions at 2 months after randomization $(\mathrm{cm})$ & $1.005(1.003-1.006)$ & $<0.0001$ \\
\hline & ETS (any tumor increase, without new metastases & $0.574(0.422-0.78 I)$ & 0.0004 \\
\hline & vs new metastases) & & \\
\hline & ETS (minor tumor shrinkage vs new metastases) & $0.7 \mathrm{II}(0.542-0.934)$ & 0.0143 \\
\hline & PFS duration (months) & $0.994(0.992-0.995)$ & $<0.0001$ \\
\hline & PFS flag (PFS censoring vs PFS event) & $0.570(0.445-0.730)$ & $<0.0001$ \\
\hline \multirow[t]{3}{*}{ TSFLT $<9$ months } & SLD of target lesions at 2 months after randomization $(\mathrm{cm})$ & $1.004(1.002-1.006)$ & 0.0002 \\
\hline & PFS duration (months) & $0.994(0.992-0.995)$ & $<0.0001$ \\
\hline & PFS flag (PFS censoring vs PFS event) & $0.566(0.416-0.770)$ & 0.0003 \\
\hline \multirow[t]{3}{*}{ PD-FLT } & SLD of target lesions at baseline $(\mathrm{cm})$ & $1.007(1.003-1.010)$ & 0.0004 \\
\hline & PFS duration (months) & $0.992(0.989-0.995)$ & $<0.000 \mathrm{I}$ \\
\hline & Time to return to baseline SLD of target lesions (months) & $1.001(1.000-1.002)$ & 0.0021 \\
\hline \multicolumn{4}{|c|}{ Patients with squamous cell histology NSCLC } \\
\hline & SLD of target lesions at 2 months after randomization $(\mathrm{cm})$ & $1.006(1.004-1.007)$ & $<0.000 \mathrm{I}$ \\
\hline & TTG (months) & $1.080(1.015-1.148)$ & 0.0143 \\
\hline & PFS duration (months) & $0.993(0.992-0.995)$ & $<0.0001$ \\
\hline & PFS flag (PFS censoring vs PFS event) & $0.474(0.35 \mathrm{I}-0.639)$ & $<0.0001$ \\
\hline
\end{tabular}

Notes: aPlease note that the HRs are not treatment effects. An HR of I.005 as in line I means that an uptake in SLD at 2 months of I0 cm increases the risk of death by $5 \%$. 'Score test.

Abbreviations: ETS, early tumor shrinkage; HR, hazard ratio; NSCLC, non-small-cell lung cancer; OS, overall survival; PD-FLT, progressive disease as the best response to first-line therapy; PFS, progression-free survival; SLD, sum of longest diameter; TSFLT, time from start of first-line therapy to randomization; TTG, time to tumor growth. 
impact of the change in target lesion size over time as a treatment effect showed that nintedanib/docetaxel significantly decreased tumor burden and decelerated tumor size compared with placebo/docetaxel in all patients with adenocarcinoma and squamous histology. The improvements in tumor burden were greatest in the adenocarcinoma PD-FLT population, comprising those patients with larger baseline tumor burden (poorest prognosis), similar to OS findings. ${ }^{13}$ In LUME-Lung 1, in adenocarcinoma patients with PD-FLT, the median OS in the nintedanib arm (9.8 months) improved by 3.5 months compared with the placebo arm (6.3 months), with a $38 \%$ reduced risk of new lesions or death. The decrease in tumor size was also greatest in this patient population, with a treatment difference of $19.7 \mathrm{~mm}$ favoring the nintedanib arm 6 months after randomization.

Among the adenocarcinoma patient populations analyzed, baseline tumor burden, as measured by the SLD of target lesions or by the number of metastatic sites, was greatest in patients with the poorest prognosis (PD-FLT, followed by TSFLT <9). However, the treatment effect of nintedanib was larger in these patients. The insensitivity toward chemotherapy observed in the PD-FLT population could represent a distinct biology of tumors that are potentially more dependent on angiogenesis and in which inhibition of angiogenesis might be more effective. Reaching and maintaining a large tumor burden may depend heavily on the development of new blood vessels to ensure high levels of oxygen and nutrients, rendering them more sensitive to the effects of antiangiogenic treatment than patients with lower tumor burden. Although nintedanib is not approved in squamous patients, we included this patient population in our analysis to determine if a meaningful treatment effect could be observed in squamous patients. Previous meta-analysis has shown that standardized uptake value, a semi-quantitative simplified measurement of tissue deoxyglucose metabolic rate, has prognostic significance for survival in NSCLC. ${ }^{24}$ Whether differences in standardized uptake values may account for the treatment effects observed here has not been evaluated, but may be a potential area of further investigation.

Decreased tumor burden, slowing increases in tumor size, and longer time without new lesions are outcomes that provide further evidence of the treatment benefit with nintedanib and suggest that further evaluation of the implications of these findings for patients with adenocarcinoma is warranted. The current analyses provide a valuable analysis and description of the antitumor effect of nintedanib that might not be evident from the response rates alone, and as such, future trials should consider evaluating tumor dynamics as a study endpoint. These analyses confirm that more aggressive tumors obtain the largest benefit from the nintedanib/ docetaxel treatment combination. Another consideration is that early assessment of tumor growth kinetics may contribute to our understanding of tumor hyperprogression in patients undergoing immunotherapy, and as such warrants further clinical evaluation. Although what decrease in tumor size represents a clinically meaningful improvement for a patient has not been established in the literature, measuring change in tumor size from a clinical perspective allows for an earlier assessment of therapeutic activity and has been correlated with survival outcomes. ${ }^{4,6-11}$

The analyses presented here are exploratory and have several limitations. Formal validation of these variables, and their potential for use as surrogate endpoints, was beyond the scope of this analysis. The study did not systematically collect imaging data post-progression on the timing and appearance of new lesions beyond PD. It should be noted that analyses only included data from selected representative lesions based on RECIST that were predefined during the trial and may not be representative of all lesions. Individual patient heterogeneity should also be considered as some SLD data may reflect the size of the primary tumor, while other data may reflect the sum of several metastatic lesions measured. A further limitation is that our analysis explored the PD-FLT and TSFLT $<9$ subgroups of adenocarcinoma patients, but the same subgroups were not studied in the patients with squamous cell carcinoma.

\section{Conclusion}

Corresponding well with the OS findings of the LUMELung 1 study, treatment with nintedanib/docetaxel resulted in trends toward decreased tumor burden, decelerated tumor size over time, and increased time to new occurrences of lesions compared with placebo/docetaxel in the overall adenocarcinoma population, with significant benefits observed for patients with the poorest prognosis due to aggressive tumor dynamics.

\section{Acknowledgments}

Statistical analysis was conducted by Hannes Buchner of Staburo $\mathrm{GmbH}$ on behalf of Boehringer Ingelheim Pharma GmbH \& Co. KG, Munich, Germany. The studies on which these analyses were based were sponsored by Boehringer Ingelheim. Analyses were conducted by Boehringer Ingelheim. Medical writing assistance, supported financially by Boehringer Ingelheim, was provided 
by Syneos Health Communications during the preparation of this manuscript.

\section{Disclosure}

Martin Reck reports personal fees from Boehringer Ingelheim, Hoffmann-La Roche, Eli Lilly, Merck Sharp \& Dohme Limited (MSD), Bristol-Myers Squibb (BMS), AstraZeneca, Celgene, Merck and Pfizer, outside the submitted work; Anders Mellemgaard reports personal fees from advisory boards from Boehringer Ingelheim, MSD, and Eli Lilly and as a speaker from Boehringer Ingelheim, outside the submitted work; Silvia Novello reports personal fees (Speaker Bureau) from MSD, BMS, Eli Lilly, AstraZeneca, and Roche, outside the submitted work. Pieter Postmus reports personal fees from advisory boards from Boehringer Ingelheim (during the conduct of the study), AstraZeneca, AbbVie, BMS, Eli Lilly, Janssen, G1 Therapeutics, MSD, Novartis, and Roche, outside the submitted work; Birgit Gaschler-Markefski is an employee of Boehringer Ingelheim; Rolf Kaiser is an employee of Boehringer Ingelheim and reports a patent issued (EP 2994125); and Hannes Buchner reports personal fees from Boehringer Ingelheim during the conduct of the study. The authors report no other conflicts of interest in this work.

\section{References}

1. Saad ED, Buyse M. Overall survival: patient outcome, therapeutic objective, clinical trial end point, or public health measure? J Clin Oncol. 2012;30(15):1750-1754.

2. Røsland GV, Engelsen AS. Novel points of attack for targeted cancer therapy. Basic Clin Pharmacol Toxicol. 2015;116(1):9-18.

3. Gerber DE, Dahlberg SE, Sandler AB, et al. Baseline tumour measurements predict survival in advanced non-small cell lung cancer. $\mathrm{Br} J$ Cancer. 2013;109(6):1476-1481.

4. Wang Y, Sung C, Dartois C, et al. Elucidation of relationship between tumor size and survival in non-small-cell lung cancer patients can aid early decision making in clinical drug development. Clin Pharmacol Ther. 2009; 86(2):167-174

5. Usuda K, Saito Y, Sagawa M, et al. Tumor doubling time and prognostic assessment of patients with primary lung cancer. Cancer. 1994;74(8): 2239-2244.

6. Claret L, Gupta M, Han K, et al. Evaluation of tumor-size response metrics to predict overall survival in Western and Chinese patients with first-line metastatic colorectal cancer. J Clin Oncol. 2013;31(17):2110-2114.

7. Giessen C, Laubender RP, Fischer von Weikersthal L, et al. Early tumor shrinkage in metastatic colorectal cancer: retrospective analysis from an irinotecan-based randomized first-line trial. Cancer Sci. 2013;104(6): 718-724.

8. Heinemann V, Stintzing S, Modest DP, Giessen-Jung C, Michl M, Mansmann UR. Early tumour shrinkage (ETS) and depth of response $(\mathrm{DpR})$ in the treatment of patients with metastatic colorectal cancer (mCRC). Eur J Cancer. 2015;51(14):1927-1936.
9. Milella M. Optimizing clinical benefit with targeted treatment in $\mathrm{mRCC}$ : "Tumor growth rate" as an alternative clinical endpoint. Crit Rev Oncol Hematol. 2016;102:73-81.

10. Modest DP, Laubender RP, Stintzing S, et al. Early tumor shrinkage in patients with metastatic colorectal cancer receiving first-line treatment with cetuximab combined with either CAPIRI or CAPOX: an analysis of the German AIO KRK 0104 trial. Acta Oncol. 2013;52(5):956-962.

11. Nozawa H, Nishikawa T, Tanaka T, et al. 'Deepness of Response' Is Associated with Overall Survival in Standard Systemic Chemotherapy for Metastatic Colorectal Cancer. Chemotherapy. 2014;60(5-6): 360-367.

12. Morgensztern D, O’Brien M, Ong T, Socinski M, Postmus P, Ko A. MA08.06 Impact of Depth of Response (DpR) on Survival in Patients with Advanced NSCLC Treated with First-Line Chemotherapy. J Thorac Oncol. 2017;12(1):S387-S388.

13. Reck M, Kaiser R, Mellemgaard A, et al. Docetaxel plus nintedanib versus docetaxel plus placebo in patients with previously treated non-small-cell lung cancer (LUME-Lung 1): a phase 3, double-blind, randomised controlled trial. Lancet Oncol. 2014;15(2):143-155.

14. Gaschler-Markefski B, Sikken P, Heymach JV, et al. Time since start of first-line therapy as a predictive clinical marker for nintedanib in patients with previously treated non-small cell lung cancer. ESMO Open. 2017; 2(1):e000102.

15. EMA. Vargatef $®$ (nintedanib) summary of product characteristics; 2016. Available from: http://www.ema.europa.eu/docs/en_GB/ document_library/EPAR_-_Product_Information/human/002569/ WC500179970.pdf. Accessed June 07, 2016.

16. Reck M, Novello S, Mellemgaard A, et al. Impact of tumor burden on the overall survival analysis of the LUME-Lung 1 study: a randomized, double-blind phase 3 trial of nintedanib (BIBF 1120) + docetaxel in NSCLC patients progressing after first-line chemotherapy. $J$ Thorac Oncol. 2013;8:O16.01(abstract)

17. Roth GJ, Binder R, Colbatzky F, et al. Nintedanib: from discovery to the clinic. J Med Chem. 2015;58(3):1053-1063.

18. Gottfried M, Bennouna J, Bondarenko I, et al. Efficacy and Safety of Nintedanib Plus Docetaxel in Patients with Advanced Lung Adenocarcinoma: Complementary and Exploratory Analyses of the Phase III LUME-Lung 1 Study. Target Oncol. 2017;12(4):475-485.

19. Tate SC, Andre V, Enas N, Ribba B, Gueorguieva I. Early change in tumour size predicts overall survival in patients with first-line metastatic breast cancer. Eur J Cancer. 2016;66:95-103.

20. Eisenhauer EA, Therasse P, Bogaerts J, et al. New response evaluation criteria in solid tumours: revised RECIST guideline (version 1.1). Eur $J$ Cancer. 2009;45(2):228-247.

21. Stein WD, Gulley JL, Schlom J, et al. Tumor regression and growth rates determined in five intramural NCI prostate cancer trials: the growth rate constant as an indicator of therapeutic efficacy. Clin Cancer Res. 2011;17(4):907-917.

22. Quartino AL, Claret L, Li J. Evaluation of tumor size metrics to predict survival in advanced gastric cancer. The 22nd of the Population Approach Group in Europe. 11-14 June, 2013; Glasgow, Scotland. Abstract 2812.

23. Girard P, Kovar A, Brockhaus B, Zuehlsdorf M, Schlichting M, Munafo A. Tumor size model and survival analysis of cetuximab and various cytotoxics in patients treated for metastatic colorectal cancer. The 22nd of the Population Approach Group in Europe. 11-14 June, 2013; Glasgow, Scotland. Abstract 2902.

24. Paesmans M, Berghmans T, Dusart M, et al. Primary tumor standardized uptake value measured on fluorodeoxyglucose positron emission tomography is of prognostic value for survival in non-small cell lung cancer: update of a systematic review and meta-analysis by the European Lung Cancer Working Party for the International Association for the Study of Lung Cancer Staging Project. J Thorac Oncol. 2010;5(5):612-619. 


\section{Publish your work in this journal}

OncoTargets and Therapy is an international, peer-reviewed, open access journal focusing on the pathological basis of all cancers, potential targets for therapy and treatment protocols employed to improve the management of cancer patients. The journal also focuses on the impact of management programs and new therapeutic agents and protocols on

patient perspectives such as quality of life, adherence and satisfaction. The manuscript management system is completely online and includes a very quick and fair peer-review system, which is all easy to use. Visit http://www.dovepress.com/testimonials.php to read real quotes from published authors.

Submit your manuscript here: http://www.dovepress.com/oncotargets-and-therapy-journal 